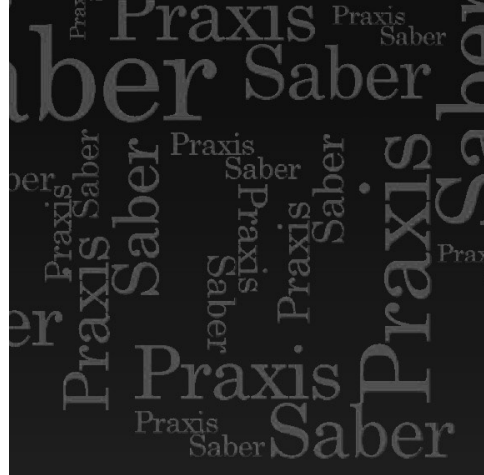

Sandra Milena Díaz Chaves

Maestría en Educación

Institución Educativa Rural

Departamental Cacicazgo

(Cundinamarca, Colombia)

mil-dicha@hotmail.com

https://orcid.org/0000-0003-0006-4154

Sandra Cecilia Arandia Rodríguez

Maestría en Educación

Institución Educativa Departamental

Integrado San Cayetano

(Cundinamarca, Colombia)

ninaceciar85@yahoo.es

https://orcid.org/0000-0001-7153-9888

Artículo de Investigación

Recepción: 11 de mayo de 2018

Aprobación: 16 de noviembre de 2018

https://doi.org/10.19053/22160159.v10.n22.2019.9319

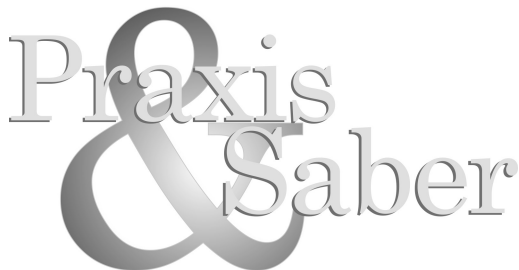

Revista de Investigación y Pedagogía Maestría en Educación. Uptc

\section{LOS ESPACIOS DE FORMACIÓN CONTINUA RESPECTO A LA ENSEÑANZA DE LENGUA ESCRITA}

\section{Resumen}

El artículo presenta el resultado del análisis de antecedentes de la investigación Espacios significativos en la formación continua de docentes en ejercicio en torno a las prácticas de enseñanza de la lengua escrita: alternativas desde la perspectiva de las comunidades de práctica, los cuales sirven para visibilizar la importancia de modelos de formación continua de docentes en ejercicio, sus características y particularidades. En este sentido, se hace un énfasis en las comunidades de aprendizaje y las comunidades de práctica, así como en los enfoques constructivista, conductista y cognitivo, para visibilizar las perspectivas y concepciones de los docentes con respecto a la enseñanza de la lengua escrita. De este proceso de indagación, se infiere que, para que surja la transformación de las prácticas de enseñanza de la lengua escrita, es importante replantear los espacios y buscar aquellos donde se permita tener en cuenta el contexto, las experiencias, las habilidades y los saberes, con el fin de encaminarlos a la construcción colectiva de conocimiento y significado, y así fortalecer el quehacer pedagógico, en particular la enseñanza de la lengua escrita, como una práctica social, al partir de hipótesis y conocimientos previos del estudiante.

Palabras claves: formación continua de docentes en ejercicio, comunidades de práctica, comunidades de aprendizaje, procesos de enseñanza-aprendizaje, lengua escrita, enfoque constructivista, enfoque conductista, enfoque cognitivo. 


\section{Continuing Training Spaces for the Teaching of Written Language}

\section{Abstract}

This article presents the result of the background analysis for the research project Significant spaces in continuing training of in-service teachers regarding practices in the teaching of written language: alternatives from the perspective of Communities of Practice. These spaces highlight the importance of continuing formation models for in-service teachers, their characteristics and specificities. In this respect, particular emphasis is placed on communities of learning and practice, as well as on constructivist, behavioral, and cognitive approaches, in order to visualize the perspectives and conceptions of teachers regarding the teaching of written language. It follows from this research process that it is important to revise such spaces to support transformation of practices in the teaching of written language. Likewise, it is necessary to find those considering contexts, experiences, skills, and knowledge, which can be geared towards the collective construction of knowledge and meaning. Pedagogical work, particularly the teaching of written language, is thus enhanced as a social practice, since it uses students' previous knowledge and hypotheses as a basis.

Keywords: continuing training of in-service teachers, communities of practice, communities of learning, teaching-learning processes, written language, constructivist approach, behaviorist approach, cognitive approach.

\section{Les Espaces de Formation Continue Pour L'ENSEIGNEMENT DE LA LANGUE ÉCRITE}

\section{Résumé}

Cet article présente le résultat de l'analyse contextuelle du projet de recherche Des espaces importants dans la formation continue des enseignants en fonction concernant les pratiques d'enseignement de la langue écrite : des alternatives dans la perspective des communautés de pratique. Ces espaces soulignent l'importance de modèles de formation continue des enseignants en fonction, leurs caractéristiques et particularités. À cet égard, un accent particulier est mis sur les communautés d'apprentissage et de pratique, ainsi que sur les approches constructiviste, comportementale, et cognitive afin de visualiser les points de vue et les conceptions des enseignants quant à l'enseignement de la 
langue écrite. Il résulte de ce processus de recherche qu'il est important de repenser les espaces pour favoriser la transformation des pratiques d'enseignement de la langue écrite. De même, il est nécessaire de trouver ceux prenant en compte les contextes, les expériences, les compétences, et les savoirs, et qui peuvent être orientés vers la construction collective du savoir et de la signification. Le travail pédagogique, particulièrement l'enseignement de la langue écrite, est ainsi renforcé en tant que pratique sociale, étant donné qu’il utilise comme base des hypothèses et des connaissances préalables de l'étudiant.

Mots-clés: formation continue des enseignants en fonction, communautés de pratique, communautés d'apprentissage, processus d'enseignement-apprentissage, langue écrite, approche constructiviste, approche comportementale, approche cognitive.

\section{Os Espaços de Formação Contínua Respeito Ao Ensino Da Língua Escrita}

\section{Resumo}

O artigo apresenta o resultado da análise de antecedentes da pesquisa Espaços significativos na formação contínua de docentes em exercício em torno às práticas de ensino da língua escrita: alternativas desde a perspectiva das comunidades de prática, os quais servem para visibilizar a importância de modelos de formação continua de docentes em exercício, suas características e particularidades. Neste sentido, se faz uma ênfase nas comunidades de aprendizagem e as de prática, assim como nos enfoques construtivista, behaviorista e cognitivo, para visibilizar as perspectivas e concepções dos docentes com respeito ao ensino da língua escrita. Deste processo de indagação, se infere que, para que surja a transformação das práticas de ensino da língua escrita, é importante reformular os espaços e procurar aqueles onde se permita ter em conta o contexto, as experiências, as habilidades e os saberes com a finalidade de encaminhá-los à construção coletiva de conhecimento e significado, e assim fortalecer o afazer pedagógico, em particular o ensino da língua escrita, como uma prática social, a partir de hipótese e conhecimentos prévios do estudante.

Palavras-chave: formação continua de docentes em exercício, comunidades de prática, comunidades de aprendizagem, processos de ensino-aprendizagem, língua escrita, enfoque construtivista, enfoque behaviorista, enfoque cognitivo. 


\section{Introducción}

En el marco de la Maestría en Educación con énfasis en Lenguaje de la Pontificia Universidad Javeriana, surge un proceso de reflexión acerca de la didáctica de enseñanza de la lengua escrita y la relación con los procesos de formación continua de maestros en ejercicio, que originó los siguientes cuestionamientos: ¿Qué alternativas de formación de docentes en ejercicio se han explorado? ¿Cuál de estas alternativas es la más potente para fortalecer los aprendizajes de los docentes respecto a la enseñanza de la lengua escrita?

Teniendo en cuenta lo anterior, se realizaron búsquedas en dos líneas de indagación: 1) propuestas de formación de docentes en ejercicio y 2) perspectivas y enfoques de enseñanza de la lengua escrita. Se analizaron cerca de 50 documentos, entre los que había libros, artículos de investigación, tesis y ponencias, elaborados entre 2001 y 2016, procedentes de Argentina, Estados Unidos, España, México, Chile, Australia, Cuba, Portugal, Venezuela y Colombia.

Como resultado se establecen dos categorías fundamentales: 1) Alternativas de formación y actualización de docentes en ejercicio, como espacio para la reflexión y la transformación de las prácticas de enseñanza, en la que se ubican aquellos documentos de investigación que hacen referencia a la formación de docentes en ejercicio respondiendo a diferentes propuestas y alternativas de capacitación y reflexión del quehacer pedagógico, por ejemplo, grupos, equipos y comunidades. 2) Enfoques, perspectivas, concepciones y conceptualizaciones de las prácticas de enseñanza de la lengua escrita, en la cual se agrupan, por un lado, las investigaciones que analizan los diferentes enfoques y perspectivas que han influenciado la enseñanza de la lengua escrita y, por el otro, las concepciones y conceptualizaciones que subyacen a la práctica docente.

\section{Alternativas de formación y actualización de docentes en ejercicio como espacio para la reflexión y transformación de las prácticas de enseñanza}

A continuación, se presenta el abordaje de la primera categoría de investigación, teniendo en cuenta algunos aspectos relacionados con las propuestas que se han utilizado a lo largo de los años para la formación docente, en las que se incluyen alternativas de formación vertical 
y de formación horizontal. En un segundo momento se realiza una descripción de las comunidades de aprendizaje [CDA] y se finaliza con la profundización en las comunidades de práctica [CoP]. Las CDA y las CoP resultan viables para crear espacios reflexivos y transformadores de las prácticas de enseñanza.

De acuerdo con esta categoría de análisis, se reconocen las necesidades de formación continua de docentes en ejercicio, donde es pertinente afirmar que la práctica docente pone al maestro en situaciones de reflexión que trascienden la mera instrucción conceptual impartida por un experto. Como lo afirma Ochoa (2005, p. 5), "[1]a formación de docentes en ejercicio [...] [requiere] frente a la formación pre-gradual y pos-gradual, ligarse a las necesidades concretas, locales y particulares porque de eso depende el éxito de la enseñanza”. En efecto, surgen dos modalidades de formación docente: formación de tipo horizontal, centrada en el trabajo en equipo, grupos o comunidades en busca de soluciones a necesidades afines desde su experiencia, conocimientos y saberes de sus integrantes, y la formación vertical, orientada por un experto que maneja cierto tipo de conocimiento para ser impartido mediante charlas, talleres, exposiciones, capacitaciones, entre otros (León, 2012). El "profesorado se apropia de conocimientos y habilidades didácticas que le permiten 'transmitir' conocimientos a sus estudiantes, resulta obsoleta y por tanto se impone necesariamente una concepción diferente de la formación docente" (González \& González, 2007, p. 3).

Formación de docentes en ejercicio: acercamiento a algunas alternativas.

Algunas de las investigaciones revisadas (González \& González, 2007; León, 2012; Meza \& Aguirre, 2001; Nóvoa, 2009; Ramírez, 2015) convergen en definir la importancia del uso de modelos de formación docente que partan del contexto real donde se desempeñen los maestros, para fortalecer la actuación del educador a través de propuestas y alternativas que permitan generar cambios en la práctica. Las investigaciones anteriormente citadas se realizaron con docentes en ejercicio de niveles de transición, básica y profesional, y partieron de las experiencias propias de dichos maestros, poniendo de relieve diferentes modalidades de formación continua, horizontales y verticales, que son orientadas al perfeccionamiento de las competencias, los saberes disciplinares y la actualización pedagógica. En consecuencia: 
Se muestra la necesidad de poner en marcha un nuevo modelo formativo no obligatorio, contextualizado a las demandas de cada centro educativo y que cuente con un asesor de apoyo cercano al aula, incluso dentro del aula. Al tiempo que no olvide la formación inicial del profesorado. (Ramírez, 2015, p. 211)

Del mismo modo, la formación de docentes se debe enmarcar dentro del contexto educativo de la práctica de cada institución, lo que permite el intercambio de saberes y la reflexión sobre el quehacer pedagógico con sus compañeros o un asesor de apoyo. De hecho, León (2012) argumenta que la formación continua tiene su principal unidad de análisis en la posibilidad constante de aprendizaje, que en sí representa el ejercicio de la profesión docente, encontrando un lugar importante en las reflexiones que se realicen de las acciones del profesorado. Dado lo anterior, León (2012) aborda dos estrategias: cadenas de aprendizaje y modelos de formación vertical y horizontal. La formación vertical se caracteriza por charlas y talleres orientados por un experto. La horizontal se basa en espacios formativos que surgen del trabajo en equipo.

En esta misma línea, Nóvoa (2009) considera otras estrategias específicas de la formación de docentes en ejercicio, como el consenso discursivo, el cual permite llegar a acuerdos entre los participantes a través de la palabra, encontrando vacíos y necesidades sobre el conocimiento; el trabajo en equipo colaborativo, entendido como aquellos procesos con intención grupal en busca de objetivos concretos; y los equipos pedagógicos, que hacen referencia al intercambio de saberes entre docentes para atender necesidades de la práctica.

Por último, otra alternativa para la formación es el taller, que "permite reafirmar la necesidad de investigar en el aula [...] en todo momento, [partiendo de] los intereses, experiencias, vivencias y conocimientos previos del grupo" (Meza \& Aguirre, 2001, p. 282), siendo entonces una alternativa de tipo vertical, que es orientada por un experto.

Comunidad de aprendizaje: un acercamiento detallado a la que podría ser la alternativa de formación de docentes en ejercicio usada con mayor frecuencia.

Por su parte, otro conjunto de investigadores (Díez-Palomar \& Flecha, 2010; Flecha \& Puigvert, 2005; González-Weil, Gómez, Ahumada, 
Bravo, Salinas, Avilés, Pérez \& Santana, 2014; Meza \& Aguirre, 2001; Padilla, Ortiz \& López, 2015; Rodríguez, 2007) y coinciden en la formación de docentes a través del trabajo en CDA, definidas como espacios que promueven la reflexión de las prácticas, donde se comparten experiencias y conocimientos colectivamente, que transforman y transcienden el ejercicio docente. Estas comunidades se caracterizan por el diálogo permanente entre los miembros de la comunidad, el análisis de problemáticas comunes y el compromiso por solucionarlas, representado en acciones colaborativas y recíprocas.

La formación continua de docentes en ejercicio mediante las comunidades de aprendizaje cuenta con la participación en charlas, conferencias, tertulias y conversatorios, que inducen a la reflexión y a la transformación de las prácticas escolares. Allí se fomenta el debate sobre la práctica. Se lleva a cabo una "fase de sensibilización en donde el docente asume responsabilidades, cede algunas y comparte otras" (Flecha \& Puigvert, 2005, p. 32). Las CDA se identifican por ser espacios donde un grupo de personas se reúnen con la intención de participar de una manera activa en la búsqueda de soluciones a dudas, incógnitas y asuntos relacionados con el contexto social y laboral. Sobre esto, Ramírez \& González (2013) afirman que las comunidades de aprendizaje "se inscriben en las teorías críticas que buscan la transformación social desde las prácticas educativas y la vida cotidiana" (p. 5). Para el tema específico de la enseñanza de la lengua escrita, las CDA han sido una alternativa de formación de docentes en ejercicio con miras a analizar y transformar las prácticas, como en el caso de la investigación realizada por Ramírez \& González (2013), quienes plantean

[un] proceso de investigación a partir de la necesidad de reflexionar sobre el quehacer pedagógico frente a los procesos de enseñanza y aprendizaje del lenguaje escrito, de manera que contribuya a mejorar el nivel de esta área de conocimiento, apoyado en las herramientas tecnológicas mediante la conformación de una comunidad de aprendizaje. (Ramírez \& González, 2013, p. 1)

En varios estudios, Padilla et al. (2015) y Díez-Palomar y Flecha (2010) confluyen en ver la comunidad de aprendizaje como un medio de participacion dialógica y social. En este sentido, las CDA se convierten en una respuesta eficiente a la hora de gestar propuestas-alternativas de formación docente, teniendo en cuenta los cambios, retos y sucesos sociales y educativos. Cabe destacar la importancia de los conocimientos 
que pueden aportar cada uno de sus miembros a la construcción colectiva (MEN, 2013). Según Díez-Palomar y Flecha (2010, p. 19), las CDA "implican a todas las personas que de forma directa o indirecta influyen en el aprendizaje".

Corresponde mencionar que la modalidad de trabajo de las comunidades de aprendizaje es flexible, en tanto existen formas de trabajo presencial y virtual. Para el caso de los trabajos de investigación anteriormente analizados, cuatro de cinco se desarrollan presencialmente; sin embargo, Padilla et al. (2015) consideran importante el trabajo en línea, dado que recoge elementos fundamentales para el desarrollo de aprendizajes, como lo son la llamada presencia social, la presencia cognitiva y la presencia docente. Por ello, sin importar si la modalidad es presencial o virtual, las CDA resultan interesantes para el abordaje de la formación docente.

\section{Comunidad de práctica: Una alternativa contemporánea de formación de docentes en ejercicio.}

Otra alternativa encontrada mientras se ahondaba en los posibles espacios de formación a docentes es la CoP, abordada por varios investigadores (Au, 2002; Ávalos, 2011; Boud \& Middleton, 2006; Bozu \& Imbernon, 2009; Fernández \& Valverde, 2013; García, Greca \& Meneses, 2008; Gómez, Rocaro, Val \& Zerillo, 2014; Rodríguez, 2007; Sanz, 2005; Sanz-Martos \& Pérez-Montoro, 2009; Vega \& Quijano, 2010; ) y definida como "grupos de personas, con distintos niveles de conocimientos, habilidades y experiencia, que se implican de un modo activo en procesos de colaboración en la resolución de problemas y construyen conocimiento tanto personal como colectivo". (García et al., 2008, pp. 440-441).

Las CoP fueron planteadas por Wenger (2001) en el siglo XX y tienen tres características propias: repertorio compartido, compromiso mutuo y empresa conjunta. En estas, la responsabilidad es de todos los integrantes que la conforman. Tienen sentido de pertenencia. Tienen fuertes vínculos con la parte social y "la participación no solo se refiere a los eventos locales de compromiso con actividades y con determinadas personas, sino también a un proceso de mayor alcance con participación activa en las prácticas de las comunidades sociales y en construir identidades" (Wenger, p. 21). Esta alternativa de formación 
docente permite tener en cuenta algunas necesidades fundamentales que emergen del proceso de enseñanza de la lengua escrita, lo que fortalece el compartir con el par las problemáticas y a la vez negociar posibles soluciones.

Las CoP pueden ser de dos modalidades: virtual y presencial. Las primeras, abordadas por diferentes autores (Fernández \& Valverde, 2013; García et al. 2008; Gómez et al., 2014; Rodríguez, 2007; Sanz, 2005), se definen como un grupo de personas con un interés mutuo que aportan y comparten información, conocimiento, experiencias, sucesos e intereses a través de una interacción continua mediada por las tecnologías de la información y la comunicación [TIC]. De hecho, Rodríguez (2007) afirma que

Una característica definitoria de una comunidad virtual en este sentido es que una persona o institución debe de ser un contribuyente a la base de conocimiento en evolución del grupo y no solamente un receptor o consumidor de los servicios o base de conocimientos del grupo. (p. 11)

Otro rasgo de las comunidades de práctica virtual es facilitar un acercamiento entre sus miembros a través de las TIC, de tal forma que factores como la distancia no alteren su funcionamiento, la participación de todos los miembros y la construcción colectiva de saberes para "gestionar contenidos intercambiados, almacenados de manera adecuada para recuperarlos de una manera fácil" (Sanz, 2005, p. 26). El uso de las TIC permite preservar la memoria colectiva de las CoP, de la misma forma que el modelo conceptual de aprendizaje colaborativo en línea posibilita la interacción entre los estudiantes y maestros, para facilitar y construir el desarrollo de capacidades, buscando la interdependencia cognitiva y social que contribuya a una dinámica horizontal (Sanz, 2005), de modo que "proporcione oportunidades para el desarrollo de habilidades sociales y comunicativas, actitudes positivas hacia las personas, cohesión grupal y construcción de relaciones sociales" (Fernández \& Valverde, 2013, p. 98).

Cabe resaltar que este tipo de espacios de formación de docentes en ejercicio, como son las comunidades de práctica virtuales, son "efectivas para la implementación de una metodología didáctica innovadora, pues está centrada en una formación en la práctica de los docentes" (García et al., 2008, p. 439), que tiene en cuenta el auge de las TIC como un instrumento para compartir experiencias en común, aunque ello pueda 
representar la pérdida de elementos importantes en la comunicación como gestos, ademanes y movimiento corporal, en tanto elementos importantes a la hora de construir significado colectivamente.

Por otro lado, la modalidad presencial de las CoP (Au, 2002; Ávalos, 2011; Boud \& Middleton, 2006; Bozu \& Imbernon, 2009; Sanz-Martos \& Pérez-Montoro, 2009; Vega \& Quijano, 2010) está centrada en espacios para la creación de conocimiento cara a cara, o diferenciada por Vega \& Quijano (2010) como un espacio de aprendizaje de los individuos participantes, donde se destaca que los niveles de conocimiento y las experiencias son diferentes en cada individuo, lo que hace que la $\mathrm{CoP}$ se convierta en un espacio potente para aprender de los otros. "Permite evidenciar la importancia de incluir actividades relevantes asociadas a prácticas reales, así como las reglas de colaboración y de distribución de las acciones en situaciones que son significativas para un grupo de personas" (Vega \& Quijano, 2010, p. 97).

Por su parte, Bozu e Imbernon (2009) hacen

una reflexión acerca de la importancia y la necesidad de crear comunidades de práctica en la universidad, como una fuente distinta de tener acceso al conocimiento, construirlo y difundirlo y como alternativa posible en el desarrollo de los programas de formación y desarrollo profesional del profesorado. (p. 2)

Consideran que las CoP permiten crear un espacio potente para la discusión, el intercambio de información y la toma de decisiones sobre posibles acciones de mejora o cambio. De igual manera, Ávalos (2011) hace referencia a la comunidad de práctica como un espacio de aprendizaje cuyo accionar influye en el contexto donde se desarrolla, dado que este grupo comparte sus conocimientos para fortalecerlos y enriquecerlos. De hecho,

Wenger (2006) [...] [afirma que] la comunidad de práctica son grupos de personas que comparten una preocupación o pasión por algo que realizan y que en la medida en que interactúan entre sí en forma regular, aprenden cómo mejorar esta actividad.(Ávalos, 2011, p. 239)

A su vez, la investigación de Bozu e Imbernon (2009) arroja la creación de una CoP presencial, virtual y reflexiva con una organización horizontal donde "un grupo de personas comparten una preocupación y un conjunto de problemas comunes acerca de la temática de la 
función pedagógica y la formación docente" (p. 8), por lo que es pertinente afirmar la importancia de la CoP como espacio de formación docente, que parte de compartir conocimientos, experiencias, saberes y habilidades, propiciar el diálogo compartido y, desde allí, construir a partir de la diversidad como proceso enriquecedor para llegar a soluciones colectivas de las problemáticas.

Por lo descrito hasta el momento, se puede afirmar que la CoP diverge de todo tipo de formación vertical, dado que es una propuesta que permite trabajar sobre una problemática en común, compartiendo experiencias, saberes y necesidades, para definir tareas, discutir, analizar y producir un nuevo conocimiento, con el propósito de mejorar el desempeño en la práctica de los participantes, lo que constituye una formación de tipo horizontal (García et al., 2008).

De hecho, las CoP son absolutamente horizontales, si se tiene en cuenta que intercambiar conocimientos, experiencias, saberes y habilidades con otras personas con perspectivas diferentes permite negociar significados, adquirir más conocimiento y promover la participación, los espacios de reflexión, el diálogo colectivo, el análisis y la solución de problemáticas que emergen de situaciones del día a día, de tal manera que se genera una serie de constructos colectivos.

Es importante destacar la investigación de Sanz-Martos y PérezMontoro (2009), quienes distinguen las comunidades frente a otros grupos y equipos de formación continua. Mencionan que los grupos y equipos "trabajan con la finalidad de alcanzar un objetivo que es lo que los une y lo que les da razón de ser" (p. 106), mientras que las comunidades se definen por el hecho mismo de compartir espacios, experiencias, conocimientos, servicios o intereses.

Además de esta diferenciación general entre equipos, grupos y comunidades, los autores mencionan que existen diferentes grupos, los de trabajo formal y los de trabajo informal, entendidos como "unas estructuras o colectivos formados por dos o más individuos que interactúan, que son interdependientes y se han articulado para cubrir objetivos particulares" (Sanz-Martos \& Pérez-Montoro, 2009, p. 106). Así mismo, los autores señalan que hay distintos tipos de equipos de trabajo: "de solución de problemas, autodirigidos, multidisciplinarios y virtuales” (Sanz-Martos \& Pérez-Montoro, 2009, 
p. 106). En los primeros, los participantes comparten ideas y dan sugerencias para mejorar los procesos de trabajo; los segundos asumen las responsabilidades de supervisores; los terceros se reúnen para llevar a cabo una tarea, intercambiar información y solucionar problemas de un mismo nivel jerárquico, y los últimos usan las TIC para reunir los participantes físicamente dispersos.

Finalmente, los autores diferencian la comunidad de práctica frente a otras comunidades, ya sean de interés o de aprendizaje, dado que en las comunidades de aprendizaje el conocimiento que "se transmite sigue estando vinculado a conceptos o materias no a maneras de hacer" (SanzMartos \& Pérez-Montoro, 2009, p. 110), mientras que las comunidades de interés se caracterizan por utilizar el internet para "compartir datos, cooperar en investigaciones e intercambiar información” (Sanz-Martos \& Pérez-Montoro, 2009, p. 110). Estas comunidades comparten un interés en común: no necesitan de un líder, comparten siempre virtualmente algo de lo que a cada participante le parece interesante.

\section{Enfoques, perspectivas, concepciones y conceptualizaciones de las prácticas de enseñanza de la lengua escrita}

La segunda categoría de análisis corresponde a las perspectivas y enfoques de las prácticas de enseñanza de la lengua escrita, las cuales dan muestra de que se tienen conceptos básicos de este proceso, ya que, en la mayoría de los casos, las escuelas y los docentes se dedican a enseñar la escritura como un proceso de codificación y su preocupación radica en la elección de un método de enseñanza que les permita lograr este objetivo (Ferreiro, 1998).

En consecuencia, la mayoría de investigaciones analizadas señalan que el proceso de enseñanza de la lengua escrita está fuertemente influenciado por las concepciones de los maestros, representadas en sus ideas, modelos, opiniones y creencias, producto de la formación recibida con respecto a los diferentes modelos y enfoques por los que ha pasado el proceso de enseñanza de la escritura (Ferreiro, 1998). En los trabajos analizados no se encuentra una respuesta para el proceso de enseñanza de la lengua escrita; por el contrario, se muestran diversas propuestas de trabajo que llevan en la mayoría de los casos a que los docentes se congreguen en diferentes modalidades de formación continua, como las comunidades de práctica, comunidades de aprendizaje, grupos 
de apoyo, entre otros, para que desde allí haya una reflexión sobre y desde sus prácticas, que les permita replantear sus concepciones y en su efecto ocurran transformaciones en las prácticas de enseñanza de la lengua escrita.

En este sentido, varias investigaciones (Araya, 2006; Baracaldo, 2007; Barba, 2004; Coll, 2005; Díaz \& Price, 2012; Duque \& Ramírez, 2014; Mujica, Díaz \& Arnáez, 2008; Reyes, Fernández-Cárdenas \& Martínez, 2013; Segura \& Kalman, 2007; Serrano, Aguirre \& Peña, 2010; Valle, 2009)destacan el análisis de diferentes concepciones, enfoques y perspectivas de la enseñanza de la lengua escrita, dentro de las que se encuentra la perspectiva de la escritura desde el enfoque constructivista, conductista y cognitivo.

\section{Perspectiva de la escritura desde el enfoque constructivista.}

Desde la perspectiva constructivista, un conjunto de estudios (Coll, 2005; Reyes et al., 2013; Segura \& Kalman, 2007; Serrano et al., 2010) abordan la enseñanza de la lengua escrita como un sistema de representación y la posicionan en un enfoque comunicativo, mediada por la interacción social. En este sentido se retoma a Segura y Kalman (2007), en la investigación La alfabetización académica mediada por usos sociales de la lengua escrita en la primaria, donde muestran un ejemplo de dos preceptos pedagógicos del enfoque comunicativo y funcional para la enseñanza del español en la primaria, el cual fue implementado en México hace más de una década. Dichos preceptos son el reconocimiento y la incorporación de usos sociales de la lengua escrita y la generación de formas diversas de interacción. "Con este acercamiento a la actividad se explora la manera en que la alfabetización académica está mediada por los usos sociales de la lengua escrita y por el repertorio de conocimientos académicos" (Segura \& Kalman, 2007, p. 42).

La enseñanza de la lengua escrita debe estar enfocada en los usos comunicativos dentro de un contexto, es decir, dar un sentido a la escritura, donde el estudiante sienta que la escritura le permite comunicarse, expresar sus ideas y sentimientos, dado que "el acceso a las prácticas sociales de la escritura depende de la posibilidad que tienen los jóvenes de participar activamente en ellas y de utilizarlas socialmente" (Serrano et al., 2010, p. 103). A partir de lo anterior, 
la enseñanza de la lengua escrita debe permitir la negociación de significados en todos los niveles de formación. "Tanto en la educación primaria como en la superior es importante que los estudiantes expresen su propia perspectiva y contrasten su visión del conocimiento" (Reyes et al., 2013, p. 105).

Es así como, a partir del enfoque constructivista, se concibe el aprendizaje de la lengua escrita como algo más que el aprendizaje del código de la escritura, pues implica la construcción de "representaciones sobre el mundo y sobre nosotros mismos y atribuir sentido a las experiencias propias y ajenas" (Coll, 2005, p. 7), lo que exige un compromiso activo y autónomo con lo escrito y una comprensión del estudiante como un sujeto que está inmerso en la cultura escrita desde su entorno familiar, social y cultural a través de anuncios publicitarios, medios de comunicación, carteles, redes sociales, entre otros, elementos que el docente debe contemplar a la hora de enseñar, teniendo en cuenta el bagaje y la experiencia con el lenguaje escrito, los conocimientos previos de los estudiantes y desde allí realizar un anclaje con su praxis.

\section{La escritura desde la perspectiva conductista.}

Un conjunto de investigaciones (Araya, 2006; Reyes et al., 2013; Valle, 2009) evidencian que la lengua escrita desde la perspectiva conductista pierde relevancia por cuanto es concebida como objeto estático, a partir de modelos como la transcripción, donde el interés del educando se deteriora en la repetición de planas sin sentido. "Los alumnos quedan excluidos de la posibilidad de expresar su propia perspectiva o de contrastar su visión del conocimiento con aquel que les es presentado en la escena académica [...] solo asimilar la voz del docente y la disciplina" (Reyes et al., 2013, p. 512). Desde esta mirada, Araya (2006) determina que "la lengua escrita es considerada un sistema externo al alumno y, por lo tanto, se enfatiza la reproducción de buenos modelos, tanto en los aspectos motores como en los de producción de textos" (p. 9). La enseñanza de la lengua escrita se restringe a la transcripción como forma de escritura, teniendo en cuenta aspectos como la caligrafía, la imitación de letras y la ortografía. Se limita a estudiar "la conducta (un fenómeno observable y medible) que se constituye en objeto de aprendizaje por medio de la repetición" (Valle, 2009, p. 13). 


\section{La escritura desde la perspectiva cognitiva.}

Al igual que el conductismo, la perspectiva cognitiva pierde objetividad, ya que concibe el lenguaje como un instrumento para transmitir y obtener información, como lo asumen Duque y Ramírez (2014, p. 30), quienes plantean que en la alfabetización "intervienen elementos perceptivos observables y mentales no accesibles al observador". Esto quiere decir que el estudiante debe desarrollar una conciencia fonológica y reconocer las palabras, simplificando la lengua escrita a una serie de sonidos; es decir, se parte del análisis de los fonemas, la asociación de sonido y letras y la progresión de conformaciones, hasta formar palabras.

Por lo anterior, resulta pertinente señalar que los procesos de enseñanza y aprendizaje de la lengua escrita en países en vías de desarrollo han sido fuertemente influenciados por tres concepciones: la constructivista, la conductista y la cognitiva "entendidas como el conjunto de saberes, creencias y explicaciones que se tienen sobre el lenguaje escrito" (Duque \& Ramírez, 2014, p. 120). Además, el proceso de enseñanza y aprendizaje de la lengua escrita está presionado por exigencias sociales e institucionales, regido por los lineamientos y estándares curriculares que se deben cumplir en aras de una educación enmarcada en las políticas de la globalización, es decir, en las líneas de la eficacia y la eficiencia, dinámicas que conllevan a que, una vez se alcanza la alfabetización, se olvide la función comunicativa y "la enseñanza se centra en la apuesta del conocimiento gramático, en una especie de ruptura, en la que el lenguaje se convierte en objeto de aprendizaje escolar" (Duque \& Ramírez, 2014, p. 120).

Ahora bien, se supone que todo niño tiene la capacidad de manejar el lenguaje escrito como una posibilidad a su alcance que le permite desenvolverse adecuadamente en una sociedad altamente alfabetizada, donde el uso de la información escrita es indispensable. Sin embargo, "diferentes investigaciones han demostrado que un porcentaje no despreciable de estudiantes presenta dificultades especialmente para producir textos, es decir, algo pasa en la educación que luego de años de escolarización se logren pobres resultados en las habilidades de escritura" (Díaz \& Price, 2012, p. 237). Desde esta posición, Barba (2004) expresa cómo se concibe la enseñanza de la lengua escrita, al evidenciar que de alguna manera se han superado los paradigmas tradicionales de la enseñanza: 
El proceso de enseñanza de la escritura constituye un proceso racional y lógico de comprensión de significados expresados por escrito, en contraposición con el aprendizaje perceptivo-motriz que considera la lectura como un descifrado de lo escrito a partir de su reconocimiento visual, y la escritura como la reproducción gráfica a partir de la destreza manual. (Barba, 2004, p. 8)

Ello quiere decir que el lenguaje escrito no corresponde a la forma de hacer la letra, los trazos, ortografía o la transcripción, sino que debe estar ligado a construir significado, ofrecer información y dar sentido a la escritura como elemento representativo del lenguaje. En concordancia con Mujica, Díaz, \& Arnáez. (2008, p. 270), "la escritura debe concebirse como un proceso interactivo, comunicativo y epistemológico”. Es aquí donde se requieren espacios que faciliten los procesos escriturales para que los estudiantes alcancen significados en la escritura. Sin embargo, para que estas transformaciones se reflejen, se debe partir de la formación continua de docentes y generar espacios donde el docente, a través de la investigación y reflexión de su propio quehacer pedagógico, pueda dar cuenta de prácticas innovadoras en torno a la enseñanza de la lengua escrita, siendo la lengua escrita un asunto que debe incidir en la escuela desde una perspectiva sociocultural.

\section{Concepciones de escritura que subyacen a la práctica docente}

Varias investigaciones (Araya, 2006; Arnáez, 2013; Bohórquez, 2016; Bowman, 2007; Castaño, 2014; Fernández-Cárdenas \& Piña-Gómez, 2014; Ferreiro, 1998; Jurado, Sánchez, Cerchiaro \& Paba, 2013; Meza \& Aguirre, 2001; Ramírez \& González, 2013; Reyes et al., 2013; Romero, Soler \& Trujillo, 2016) se concentran en la revisión de las concepciones y conceptualizaciones de escritura que subyacen a la práctica docente, entendidas como un "conjunto de ideas, modelos, opiniones, creencias o perjuicios” (Castaño, 2014, p. 13).

Estas ideas o perspectivas de los docentes tienen que ver con su proceso de formación, las percepciones del docente con respecto a la enseñanza de la escritura, cómo aprendió esta práctica, cuáles son sus experiencias personales con respecto a la escritura y para qué cree que sus estudiantes requieren este aprendizaje. (Castaño, 2014, p. 4)

El proceso de enseñanza de la lengua escrita está fuertemente influenciado por las concepciones que sobre esta tiene el profesor, muestra de ello es que, si bien muchos docentes afirman que la 
escritura hace parte de preparar a los alumnos para desenvolverse en su contexto social, siguen usando la copia, el dictado o la transcripción para su enseñanza (Castaño, 2014). Así mismo, Bohórquez (2016) concluye que, en el proceso de enseñanza-aprendizaje de la lengua escrita, muchas veces subyacen concepciones teóricas que otorgan un papel pasivo al estudiante y que centran su atención en la gramática, lo que conlleva a orientar las prácticas docentes y a comprender la lengua escrita como un simple conjunto de habilidades motoras. Por las razones anteriores, es pertinente que, desde la escuela, "el currículo, los alumnos, la motivación, los enfoques metodológicos, las estrategias didácticas y los materiales instruccionales [sean] importantes al enseñar lengua, [pero] es el docente el que ocupa un papel preponderante en la interacción docente-alumno-objeto de estudio" (Arnáez, 2013, p. 2).

Otras de las conceptualizaciones que subyacen a la práctica docente son las decisiones metodológicas, la manera de presentar las letras individuales - ¿hay que dar el nombre o el sonido? - y el orden como se presentan las palabras, lo que implica una secuencia de lo fácil a lo difícil.
La lengua escrita es un objeto de uso social, con una existencia social y no meramente escolar cuando los niños habitan un ambiente urbano, encuentran escritura en todas partes (letreros, envases comerciales, propagandas, carteles, en la televisión, etc.), en el mundo circundante están las letras no en un orden sino con la frecuencia que cada una de ellas tiene en la escritura de la lengua. (Ferreiro, 1998, p. 28)

En este sentido, investigadores de la línea sobre concepciones y conceptualizaciones que subyacen a la práctica docente (Araya, 2006; Bowman, 2007; Jurado et al., 2013; Reyes, et al., 2013) asumen, desde una perspectiva sociocultural, la necesidad de fomentar en la enseñanza de la lengua escrita "prácticas de lectura y escritura que recuperen el genuino sentido social, dialógico e identitario de toda escritura" (Reyes, et al., 2013, p. 512). Aquí es importante preguntarse por el papel del docente, frente a lo cual Bowman (2007) afirma:

El docente se constituye en un mediador en este proceso de aprendizaje de los sujetos en tanto bisagra frente al conocimiento, echando mano a la precariedad de la disponibilidad objetiva con la que cuenta. [...] proceso que ha de permitirle al sujeto reconocerse a sí mismo como aprendiz, en los conocimientos que se va apropiando, construyendo, en las posiciones que va elaborando, asumiendo, y en los instrumentos mediante los que accede a ellos. (p. 112) 
El rol del docente en el proceso de enseñanza y aprendizaje es muy amplio, pues por un lado debe tener en cuenta los conocimientos previos de los estudiantes sobre el proceso, y por otro lado, la articulación con el objeto de estudio: "[E]s indispensable que el docente maneje con propiedad el objeto de conocimiento que deberán aprender sus alumnos" (Araya, 2006, p. 10). Teniendo en cuenta el contexto social, cultural y familiar en el cual se desempeña, el sentido del docente en el aula de clases "implica que las prácticas pedagógicas superen el papel del maestro como transmisor de conocimiento, pues un conocimiento no puede transmitirse entre una persona y otra; lo que se transmite son representaciones que producen en el destinatario otras representaciones" (Jurado et al., 2013, p. 20).

\section{Conclusiones}

Las futuras prácticas de los docentes en formación continua planteadas desde una perspectiva sociocultural, permiten que se formen comunidades de docentes que compartan sus prácticas pedagógicas y busquen solución a problemáticas desde sus experiencias, saberes, conocimientos y habilidades. Así, se fortalecerá la enseñanza de la lengua escrita y se comprenderá como un sistema de representación, de forma que se incluya al niño como un sujeto cognoscente, interpretativo, propositivo, pensante y contructor de conocimiento.

En este caso, el docente es un mediador o guía del desarrollo intelectual, integral y personal del estudiante. Mediante diferentes modelos estratégicos pertinentes, le permitirá al educando desarrollar actividades escritoras. Por esta razón, es de vital importancia la reflexión y la transformación desde sus propias prácticas, donde se analicen los métodos de enseñanza, se piense en qué se enseña, cómo se enseña y para qué se enseña, ya que la adquisición de la lengua escrita va más allá del conocimiento de letras, en tanto el uso de recursos que disponen los docentes y cómo se utilizan como mediadores en el proceso. La modalidad de interacción promovida en el desarrollo de la clase podría estar favoreciendo el encuentro del alumno con la cultura escrita, como un logro cognitivo y, a su vez, social.

En síntesis, después del análisis de los diferentes espacios de formación continua de docentes en ejercicio, se puede decir que la comunidad de práctica se presenta como una alternativa potente para aquellos 
que busquen profundizar en la enseñanza de la lengua escrita como una práctica sociocultural; lo anterior, teniendo en cuenta que las CoP permiten el trabajo mancomunado, discutido, compartido y transformativo que emerge de la práctica docente, al compartir experiencias, habilidades, saberes y conocimientos para la solución de problemáticas propias de la enseñanza de la escritura y la construcción colectiva de conocimientos, como condición para poner en marcha tareas concretas en el aula.

Se considera que la formación continua es fundamental para la reflexión y la transformación de las prácticas de enseñanza de la lengua escrita, mediante espacios que permitan la interacción entre pares, el compartir con el otro, discutir la cotidianidad y expresar puntos de vista que surgen de las prácticas cotidianas, todo ello teniendo en cuenta los diferentes contextos, los niveles de enseñanza y los valores que están presentes dentro y fuera del aula de clase, tomando conciencia del quehacer pedagógico como forma de construcción de conocimiento, identidades y significados personales y colectivos.

\section{Referencias}

AraYA, L. (2006). ¿Qué nos pasa en escritura? Hipótesis sobre los problemas en la enseñanza de la lengua escrita. Lectura y Vida, 28(1), 6-15. Recuperado de http://www.lecturayvida.fahce.unlp.edu.ar/numeros/ a28n1/28_01_Araya.pdf

Arnáez, P. (2013). La enseñanza de la lengua desde la perspectiva del docente. Paradigma, 34(2), 1-22. Recuperado de http://www.scielo.org.ve/ scielo.php?script $=$ sci_arttext\&pid $=$ S1011-22512013000200002

Au, K. (2002). Communities of practice, engagement, imagination and alignment in research on teacher education. Journal of Teacher Education, 53(3), 222227. Recuperado de https://journals.sagepub.com/doi/pdf/10.1177/0 022487102053003005. https://doi.org/10.1177/0022487102053003005

Ávalos, B. (2011). El liderazgo docente en comunidades de práctica. Educar, 47(2), 237-252. Recuperado de https://www.researchgate.net/ publication/277045051_El_liderazgo_docente_en_comunidades_de_ practica

Baracaldo, M. (Coord.). (2007). Investigación de los saberes pedagógicos Bogotá: Ministerio de Educación. Recuperado de https://www. mineducacion.gov.co/1621/articles-345504_anexo_13.pdf 
BARBA, L. (2004). La enseñanza de la lengua escrita en la educación básica a través de sus programas y modelos pedagógicos. Balance y perspectivas. Perfiles Educativos, 26(103), 1-12. Recuperado de http://www.scielo. org.mx/scielo.php?script=sci_arttext\&pid=S0185-26982004000200003

BoHórquez, C. (2016). Movilización de las concepciones docentes sobre la enseñanza de la escritura en básica primaria (Tesis de maestría, Universidad Distrital Francisco José de Caldas, Bogotá, Colombia). Recuperado de http://repository.udistrital.edu.co/ bitstream/11349/2761/2/BohorquezCecilia2016.pdf

Boud, D., \& Middleton, H. (2006). Learning from others at work: communities of practice and informal learning. Journal of Workplace Learning, 15(5), 194-202. Recuperado de https://www.researchgate. net/publication/240260284_Learning_from_others_at_work_ Communities_of_practice_and_informal_learning

Bowman, M. (2007). Mediación docente y construcción de la lengua escrita en procesos de alfabetización de jóvenes y adultos. Cuadernos de Educación, 5(5), 205-219. Recuperado de https://revistas.unc.edu.ar/ index.php/Cuadernos/article/view/729/689

Bozu, Z., \& Imbernon, F. (2009). Creando comunidades de práctica y conocimiento en la universidad: una experiencia de trabajo entre las universidades de lengua catalana. Revista de Universidad y Sociedad del Conocimiento, 6(1), 1-10. Recuperado de https://www.raco.cat/index. $\mathrm{php} /$ RUSC/article/download/129746/179181

Castaño, A. (2014). Prácticas de escritura en el aula: Orientaciones didácticas para docentes. Bogotá: Ministerio de Educación Nacional - Cerlalc Unesco. Recuperado de http://aprende.colombiaaprende.edu.co/sites/ default/files/naspublic/2_practicas_de_escritura_en_el_aula.pdf

Coll, C. (2005). Lectura y alfabetismo en la sociedad de la información. Uoc Papers, Revista sobre la sociedad del conocimiento, 1, 2-12. Recuperado de https://www.researchgate.net/publication/28093086_Lectura_y_ alfabetismo_en_la_sociedad_de_la_informacion

VAlle Del, L. (2009). Enseñanza de la lengua escrita en la primera etapa de la Escuela Básica, Estudio de caso descriptivo sobre unas educadoras venezolanas (Tesis doctoral, Universidad Autónoma de Barcelona, Barcelona, España). Recuperado de www.tdx.cat/ bitstream/10803/4683/1/lvr1de3.pdf

DíAz, C. \& Price, M. F. (2012). ¿Cómo los niños perciben el proceso de la escritura en la etapa inicial? Estudios Pedagógicos, 38(1), 215-233. Recuperado de 
https://scielo.conicyt.cl/scielo.php?script=sci_abstract\&pid=S071807052012000100013\&lng=en\&nrm=iso\&tlng $=$ es

Díez-Palomar, J., \& Flecha, R. (2010). Comunidades de aprendizaje: un proyecto de formación social y educativa. Revista Interuniversitaria de Formación del Profesorado, 24(1), 19-30. Recuperado de https://www. redalyc.org/articulo.oa?id $=27419180002$

Duque, A., \& Ramírez, M. (2014). Concepciones de enseñanza del lenguaje escrito del maestro del primer ciclo de educación básica (Tesis de maestría, Universidad Tecnológica de Pereira, Pereira, Colombia). Recuperado de http://repositorio.utp.edu.co/dspace/bitstream/ handle/11059/4626/372634D946.pdf;jsessionid=F2556F2B00C108A02 A3143FFAD07DF38? sequence $=1$

Fernández, M., \& Valverde, J. (2013). Comunidades de práctica: un modelo de intervención desde el aprendizaje colaborativo en entornos virtuales. Comunicar, 21(42), 97-105. Recuperado de https://www. revistacomunicar.com/index.php?contenido $=$ detalles\&numero $=42 \&$ ar ticulo $=42-2014-09$

Fernández-CÁrdenas, J., \& Piña-Gómez, L. (2014). El oficio del escritor académico: un portal para promover el uso de la lengua escrita como práctica social. Revista mexicana de investigación educativa, 19(60), 187212. Recuperado de http://www.scielo.org.mx/scielo.php?script=sci_ar ttext\&pid=S1405-66662014000100009

Ferreiro, E. (1998). Alfabetización: teoría y práctica. Argentina. Siglo Veintiuno editores.

Flecha, R., \& Puigvert, L. (2005). Formación del profesorado en las comunidades de aprendizaje. Revista Colombiana de Educación, 48, 12-36. Recuperado de http://revistas.pedagogica.edu.co/index.php/ $\mathrm{RCE} /$ article/view/7715/6217

García, J., Greca, I., \& Meneses, J. (2008). Comunidades virtuales de práctica para el desarrollo profesional docente en enseñanza de las ciencias. Revista Electrónica de Enseñanza de las Ciencias, 7(2), 439-462. Recuperado de http://reec.uvigo.es/volumenes/volumen7/ART10_ Vol7_N2.pdf

Gómez, S., Rocaro, S., Val, A., \& Zerillo, A. (2014). Comunidad de práctica docente: Contribución a la construcción de saber. Revista Contextos de Educación, 17, 15-24. Recuperado de http://hum.unrc.edu.ar/ publicaciones/contextos/articulos/vol17/pdf/02.pdf 
GonzÁlez, R., \& GonZÁlez, V. (2007). Diagnóstico de necesidades y estrategias de formación docente en las universidades. Revista Iberoamericana de Educación, 43(6), 1-14. Recuperado de https://rieoei.org/RIE/article/ view $/ 2365$

González-Weil, C., Gómez, M., Ahumada, G., Bravo, P., Salinas, E., Avilés, D., Pérez, J. L., \& Santana, J. (2014). Principios de desarrollo profesional docente construido por y para profesores de ciencias: una propuesta sustentable que emerge desde la indagación de las propias prácticas. Estudios Pedagógicos, 40, 1-23.

https://doi.org/10.4067/S0718-07052014000200007

Jurado, F., Sánchez, L., Cerchiaro, E., \& Paba, C. (2013). Práctica pedagógica y lengua escrita: una búsqueda de sentido. Folios, 37, 17-25. Recuperado de http://www.scielo.org.co/pdf/folios/n37/n37a02.pdf

LEÓn, Z. (2012). Modelo pedagógico de formación permanente de los docentes en el proceso docente- educativo-productivo y de servicio en los institutos universitarios de tecnología. Didasc@lia: Didáctica y Educación, 3(1), 59-83. Recuperado de https://dialnet.unirioja.es/ descarga/articulo/4228381.pdf

MEN. (2013). Sistema Colombiano de Formación de Educadores y Lineamientos de Política. Bogotá: Imprenta Nacional de Colombia.

Meza, N., \& Aguirre, R. (2001). Transformar la práctica pedagógica de la lengua escrita en el aula propuesta para la formación de docentes de educación preescolar. Educere, 5(15), 275-283. Recuperado de https:// www.redalyc.org/articulo.oa?id $=35651503$

Mujica, B., Díaz, B., \& Arnáez, P. (2008). Concepción de escritura en los libros de texto de educación básica. Revista de Pedagogía, 29(85), 269291. Recuperado de http://www.scielo.org.ve/scielo.php?script=sci_art text\&pid=S0798-97922008000200004

NóvoA, A. (2009). Para una formación de profesores construida desde la profesión. Revista de Educación, 350, 203-218. Recuperado de http:// www.revistaeducacion.mec.es/re350/re350_09.pdf

OchOA, M. (2005). Al tablero, periódico de un país que educa y que se educa. Recuperado de http.//www.mineducación.gov.co/1621/article-89943. html

Padilla, S., Ortiz, L., \& López, C. (2015). Comunidades de aprendizaje en línea. Análisis de las interacciones cognitivas docentes y afectivas. 
Apertura, 7(1), 1-18. Recuperado de https://www.redalyc.org/articulo. oa?id $=68838021008$

Ramírez, A. (2015). La formación del profesorado de educación primaria ante las competencias básicas. Revista Electrónica Interuniversitaria de Formación del Profesorado, 18(3), 199-214. Recuperado de http:// revistas.um.es/reifop/article/view/193811

Ramírez, A., \& GonzÁLez, P. (2013). La transformación de la enseñanza y el aprendizaje del lenguaje escrito en un entorno bimodal a través de una comunidad de aprendizaje. Recuperado de https://docplayer. es/72262998-La-transformacion-de-la-ensenanza-y-el-aprendizaje-dellenguaje-escrito-en-un-entorno-bimodal-a-traves-de-una-comunidadde-aprendizaje-1.html. https://doi.org/10.6018/reifop.18.3.193811

Reyes, S., Fernández-Cárdenas, J., \& Martínez, R. (2013). Comunidades de blogs para la escritura académica en la enseñanza superior. Un caso de innovación educativa en México. Revista Mexicana de Investigación Educativa, 18(57), 507-535. Recuperado de http://www.scielo.org.mx/ scielo.php?script $=$ sci_arttext\&pid $=$ S1405-66662013000200009

Rodríguez, J. (2007). Comunidades virtuales, práctica y aprendizaje: elementos para una problemática. Teoría de la Educación. Educación y Cultura en la Sociedad de la Información, 8(3), 6-22. Recuperado de https://www. redalyc.org/articulo.oa?id $=201017307002$

Romero, C., Soler, S., \& Trujillo, M. (2016). Uso de mediaciones pedagógicas con TIC en el fortalecimiento de la escritura inicial. (Tesis de maestría, Pontificia Universidad Javeriana de Bogotá).

SANZ, S. (2005). Comunidades de práctica virtuales: acceso y uso de contenidos. Revista de Universidad y Sociedad del Conocimiento, 2(2), 26-35. Recuperado de https://dialnet.unirioja.es/descarga/articulo/1331910. pdf

Sanz-Martos, S., \& Pérez-Montoro, M. (2009). Conocimiento colaborativo: las comunidades de práctica y otras estrategias organizacionales. Ponencia presentada en el Congreso Nuevas perspectivas para la difusión y organización del conocimiento. Valencia, España.

Segura, M., \& Kalman, J. (2007). La alfabetización académica mediada por usos sociales de la lengua escrita en la primaria. Lectura y Vida, 28(4), 40-49. Recuperado de http://www.lecturayvida.fahce.unlp.edu.ar/ numeros/a28n4/28_04_Segura.pdf 
Serrano, S., Aguirre, R., \& Peña,J. (2010). Pensamiento del profesory acceso a la cultura escrita. Letras, 52(83), 103-132. Recuperado de http:/ /www.scielo. org.ve/scielo.php?script=sci_arttext\&pid=S0459-12832010000300004

Vega, M., \& Quijano, Á. (2010). Comunidades de práctica y alfabetización informacional. Ibersid, 4, 93-103. Recuperado de http://ibersid.eu/ojs/ index.php/ibersid/article/view/3878

Wenger, E. (2001). Comunidades de práctica. Aprendizaje, significado e identidad. Barcelona: Paidós. 